DIW BERLIN

Discussion

Papers
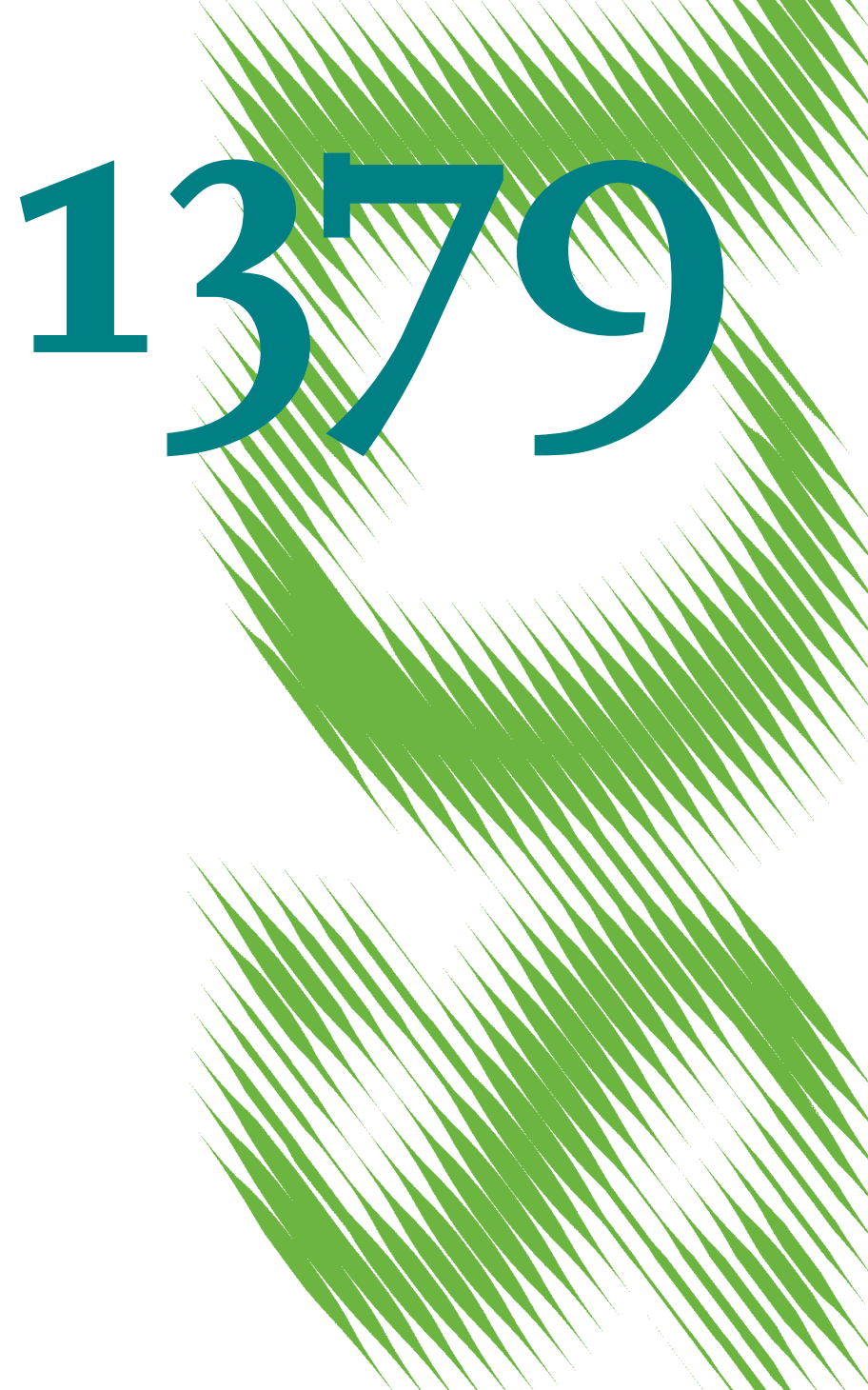

National-Strategic Investment in

European Power Transmission Capacity 
Opinions expressed in this paper are those of the author(s) and do not necessarily reflect views of the institute.

IMPRESSUM

(C) DIW Berlin, 2014

DIW Berlin

German Institute for Economic Research

Mohrenstr. 58

10117 Berlin

Tel. $+49(30) 89789-0$

Fax +49 (30) $89789-200$

http://www.diw.de

ISSN print edition $1433-0210$

ISSN electronic edition 1619-4535

Papers can be downloaded free of charge from the DIW Berlin website:

http://www.diw.de/discussionpapers

Discussion Papers of DIW Berlin are indexed in RePEc and SSRN:

http://ideas.repec.org/s/diw/diwwpp.html

http://www.ssrn.com/link/DIW-Berlin-German-Inst-Econ-Res.html 


\title{
National-strategic investment in European power transmission capacity*
}

\author{
Daniel Huppmann ${ }^{\mathrm{a}}$, Jonas Egerer ${ }^{\mathrm{a}, \mathrm{b}}$

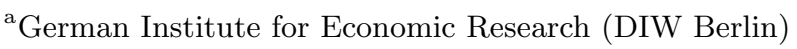 \\ Mohrenstraße 58, 10117 Berlin, Germany \\ ${ }^{\mathrm{b}}$ Berlin University of Technology, Workgroup for Infrastructure Policy \\ Straße des 17. Juni 135, 10623 Berlin, Germany \\ dhuppmann@diw.de, je@wip.tu-berlin.de
}

May 7, 2014

\begin{abstract}
The transformation of the European energy system requires substantial investment in transmission capacity to facilitate cross-border trade and to efficiently integrate renewable energy sources. However, network planning in the $\mathrm{EU}$ is still mainly a national prerogative. In contrast to other studies aiming to identify the pan-European (continental) welfare-optimal transmission expansion, we investigate the impact of zonal planners deciding on network investment strategically, with the aim of maximizing the sum of consumer surplus, generator profits and congestion rents in their jurisdiction. This reflects the inadequacy of current mechanisms to compensate for welfare re-allocations across national boundaries arising from network upgrades.

We propose a three-stage equilibrium model to describe the Nash game between zonal planners (i.e., national governments, regulators, or system operators), each taking into account the impact of network expansion on the electricity spot market and the resulting welfare effects on the constituents within her jurisdiction. Using a four-node sample network, we identify several Nash equilibria of the game between the zonal planners, and illustrate the failure to reach the first-best welfare expansion in the absence of an effective compensation mechanism.
\end{abstract}

Keywords: electricity transmission, network expansion, Generalized Nash equilibrium (GNE), mixed-integer equilibrium problem under equilibrium constraints (MI-EPEC)

JEL Codes: L51, C61, C72

${ }^{*}$ The authors would like to thank Sauleh Siddiqui and Franziska Holz for many valuable comments. Financial support by Energie-Control Austria (ECA) for D. Huppmann within the special series "10 years of e-control" is gratefully acknowledged. The views expressed in this article should not be considered as a statement regarding the views of ECA. 


\section{Introduction}

The creation of a well-integrated Internal Energy Market (IEM, EC, 2012) and the decarbonisation of the European electricity sector require both a paradigm shift within the generation portfolio as well as a significant expansion of the (cross-border) power transmission system. The switch to renewable power generation is fundamental to meeting European climate goals (EU Road Map 2050, EC, 2011); however, wind and solar are inherently volatile and the most effective sites are often not located close to cities and industrial regions. As a consequence, the transmission system must be strengthened to ensure the effective and efficient integration of these energy sources. Due to the national scope of planning electricity systems for most of the $20^{\text {th }}$ century, additional cross-border transmission capacity is required in particular to achieve these goals.

There are several recent applied studies that aim to determine the optimal network investment plans to support such a decarbonisation of the electricity sector (e.g. Egerer et al., 2013a; Tröster et al., 2011). These models take a long-term view (i.e., until the year 2050), and they typically use a pan-European welfare maximization approach, where all investment decisions are taken by a benevolent central planner. In contrast, the European Ten-Year Network Development Plan (TYNDP, ENTSO-E, 2013) only covers investments over the following decade. In contrast to the other studies mentioned, the TYNDP is not derived from an explicit welfare-maximization rationale. Instead, it lists transmission expansion projects as planned by the individual transmission system operators (TSO) and agreed within the European Network of Transmission System Operators for Electricity (ENTSO-E), the TSO's trade association, following a public stakeholder process. Some key projects for transEuropean energy infrastructure are labelled as projects of common interest by the European Commission and therefore have access to additional financial support (EC, 2013). However, all these studies and political documents neglect that transmission investments may have a strong impact on welfare distribution both across national borders and among different stakeholders: generators, consumers, and TSOs.

Network expansion is still a national prerogative in Europe, both regarding planning and financing. Grid investments are highly capital-intensive and, once built, constitute a "lock-in" regarding the grid topology for many decades to come. Funding for grid upgrades is usually guaranteed by the national regulator, who allows the TSO to levy network usage fees to recover expansion costs. If the income earned by the TSO from usage tariffs and congestion rents exceeds the approved funding base, the surplus must be used to either maintain and guarantee availability of the existing grid, invest in transmission capacity upgrades, or to lower usage fees (EC, 2009).

Due to the nature of electricity flows in an integrated network, beneficiaries of transmission investment may be located in a different jurisdiction than those bearing the costs. National governments, regulators and/or the TSOs may then be reluctant to invest if the benefits accrue elsewhere, unless an appropriate compensation mechanism is in place. Furthermore, any analysis of the power market is particularly complicated due to the specific characteristics of electricity transmission: if one line between two zones is expanded, the changes in power flow patterns may adversely affect other TSOs due to an increase of loop flows or a reduction of effectively available transmission capacity along other lines.

Egerer et al. (2013b) discuss the welfare implications for different topologies of the North and Baltic Seas offshore connectors. They show a significant re-allocation of rents, both across jurisdictions, as well as between the different stakeholder groups. This indicates that the cost allocation of network investment is of paramount importance (cf. Buijs et al., 2010). In principle, the beneficiaries of any network upgrade should bear the costs. With this in mind, the EU has introduced the Inter-TSO compensation mechanism (ITC, EC, 2010), intended to remunerate TSOs for transit flows and grid upgrades of regional importance.

Theoretically, an appropriate allocation of benefits and costs through side payments 
would result in a grand coalition and yield a welfare-optimal expansion on the European level. There is a string of scientific literature examining various allocation methods based on cooperative game theory (Nylund, 2013, Gately, 1974). However, quantifying re-allocation of welfare might prove impossible due to issues of measurement; for instance, one may debate whether the no-investment welfare allocation should serve as a reference (i.e., the status quo), or a hypothetical case of unilateral investment. We believe that this difficulty is one of the reasons why the ITC is still mainly used for compensation of transit flows (where a reference can be more easily established and the monetary transfers involved are fairly small), rather than for compensation of actual network upgrades (cf. Buijs et al., 2010). The current annual budget dispensed through the ITC is around 100 million $€$ (Rüster et al., 2012, p. 25ff); the investment volume planned according to the TYNDP is more than 100 billion $€$ (ENTSO-E, 2013 ) over the next decade 1

So far, we have argued specifically with the European situation in mind; nevertheless, Buijs et al. (2010) point out that while the institutional setting in the US power markets differs from Europe in many aspects, the same underlying conundrum is present in renewables support and network upgrades between different states as well as between regional system operators such as the Pennsylvania-Maryland-New Jersey Interconnection (PJM) and the Midwest ISO (MISO).

Following this train of thought, we ask whether zonal planners would alter their transmission capacity investment if they were only concerned with national welfare, rather than the welfare of the entire region. We use the term "national-strategic" to differentiate our work from other studies that treat generators as strategic players (e.g., Zerrahn and Huppmann, 2014, Pozo et al., 2013, Schröder et al., 2013; Neuhoff et al., 2005), as well as from the literature that treats one TSO responsible for the entire network area as the strategic player (e.g., Rosellón and Weigt, 2011; Léautier and Thelen, 2009). In contrast to these articles, we assume that the strategic players in the game are zonal planners such as national governments, regulators, or TSOs in charge of a certain network area: they anticipate the effects of network expansion within their jurisdiction on the welfare allocation. They may thus have an incentive to withhold line upgrades or over-invest compared to the welfare-optimal expansion plan in order to induce a shift of rents towards stakeholders in their zone. This distortion of investment may impede the effective integration of the European power market and the efficient shift to a power system based on renewable energy sources.

The next section will briefly present the theory of network formation and discuss two previous models similar to our research question. Section 3 describes the three-stage model depicting the national-strategic investment game, while Section 4 provides the mathematical formulation. This part also extensively discusses the problems of solving multi-level games and illustrates how our approach deals with them. Section 5 provides a numerical example of the three-stage model to illustrate the impact of strategic network planning; Section 6 concludes and suggests potential avenues for further research.

\section{Network formation and strategic zonal planners}

The question of how networks are formed when some players act strategically and have to agree whether or not to build a link between them is not new in the economics and game theory literature. However, to date no canonical approach exists to solve such games (cf. Bloch and Jackson, 2007, 2006). To further complicate the research question which we tackle in this work, the size of the link in electricity networks (i.e, capacity of the power line) is of paramount importance for resulting flows and nodal prices. We therefore opt for the most straightforward game structure: zonal planners strategically decide only on the

\footnotetext{
${ }^{1}$ Of course, only a fraction of the investment costs need to be financed through a pan-European fund; most line upgrades are viable from a national perspective alone. Nevertheless, the discrepancy is significant.
} 
upgrades of lines within their jurisdiction. Cross-border lines are decided by a supra-national planer. This allows us to sidestep, for the time being, the question of how to properly model negotiation on links between zones, while we are still able to focus on the impact of strategic zonal planners. We formulate a methodology to identify Nash equilibria and quantify the welfare "loss" due to strategic behaviour on the network planning level ${ }^{2}$

A similar research question was tackled by Daxhelet and Smeers (2007), albeit their focus lies on network usage tariffs: the authors compare several proposals discussed within the Florence Regulatory Forum and discuss the different implementations of supra-national coordination. They propose a two-stage model in which national regulators set network usage tariffs for generators (G-component) and consumers (or load, hence L-component) in their jurisdiction, aiming to maximize welfare (defined as consumer surplus, generator profits and congestion rent) in their zone. In their model, the regulators play a Nash game, which forms the upper level of the model, while the spot market forms the lower level and takes the network usage tariffs in each zone as given. Mathematically, each regulator faces a mathematical program under equilibrium constraints (MPEC); the equilibrium constraints represent the lower-level optimization problem. Since several regulators interact simultaneously, the entire problem is an equilibrium problem under equilibrium constraints (EPEC), in particular a multi-leader-single-follower game (cf. Kulkarni and Shanbhag, 2013). The network in this work is treated as exogenously given.

In contrast, Buijs and Belmans (2011) focus on network upgrades; they compare the supra-national welfare-optimal investment to two other approaches: first, a setup where the planner responsible for one zone decides on investment in the entire network seeking to maximize domestic welfare comprising consumer surplus, generator profits and congestion rents less investment costs. Similar to the work by Daxhelet and Smeers, the zonal planner takes into account the impact of her decisions on the spot market, which forms the lower level; it is agnostic with respect to the welfare effects in other zones.

As a second model, Buijs and Belmans introduce a variation of the supra-national planner model, which they refer to as Pareto planner; they add explicit constraints that the aggregate welfare in each zone must not be reduced due to investment relative to the status quo. Both the zonal planner and the Pareto planner solve an MPEC; however, Buijs and Belmans do not actually compute Nash equilibria between the zonal planners. Their results only serve as upper bounds: how much could a zone benefit at most if all transmission investment was carried out in the most beneficial way for this particular zone. Similarly, their Pareto planner approach exhibits an inconsistency: it is only constrained relative to the status quo. As already pointed out, this may not be adequate if a zonal planner can unilaterally improve domestic welfare, for instance by expanding a line which is entirely within her jurisdiction.

Buijs and Belmans (2011) use genetic algorithms to solve each MPEC; it is not straightforward how this could be generalized to obtain a Nash equilibrium between the zonal planners. Two approaches to solve such EPEC-type problems are most commonly used in the literature: enumeration discretizes the strategy space for each strategic player; the lower-level optimization problem is then solved for each possible strategy of the upper-level game, and the pay-offs as well as deviation incentives for each player are determined ex-post. Such an approach is used by Egerer and Nylund (2014). The problem when implementing this approach in practice comes from the difficulty of determining a suitable discretization, and a general issue of scalability to solve large-scale problems.

In contrast to enumeration, diagonalization describes an iterative algorithm: each MPEC is solved consecutively, holding the decisions variables of the rivals fixed. This approach is sometimes referred to as a variant of the "Gauss-Seidel" algorithm. While it does work in certain applications, convergence to a stable Nash equilibrium is not guaranteed. In particular, several Nash equilibria may exist, and it is not clear whether diagonalization

\footnotetext{
${ }^{2}$ To be precise, it is not a welfare loss, but a failure to reap all welfare gains from network investment and a more efficient and integrated spot market.
} 
would identify all solutions; more importantly, one can usually make no informed statement whether other equilibria may exist; in particular, a Pareto improvement ay be possible (i.e., a new equilibrium where at least one player is strictly better off, and no player has a worse payoff than in the previously found equilibrium). We discuss this in more detail below. In the following section, we present a closed-form expression of the game between zonal planners, as well as an iterative algorithm to determine a number of Nash equilibria ranked according to a welfare metric.

\section{A three-stage equilibrium problem}

We propose a three-stage model to describe the Nash game between zonal planners; Table 1 illustrates the stages, players and decision variables of the game. We solve this game by backward induction; therefore, we present the structure from the bottom stage to the top-level player. We first only describe the model stages and players; the mathematical formulation is presented in the subsequent section.

\begin{tabular}{c|cc} 
Stage & Player & Decision Variable \\
\hline \hline I & $\begin{array}{c}\text { Supra-national planner } \\
\text { seeks to maximize welfare in the entire region } \\
\text { less investment cost }\end{array}$ & $\begin{array}{c}\text { cross-border network investment } \\
\text { II }\end{array}$ \\
\hline III & $\begin{array}{c}\text { Nash game between Zonal planners } \\
\text { seeks to maximize zonal welfare } \\
\text { less domestic investment cost }\end{array}$ & $\begin{array}{c}\text { domestic network investment } \\
\text { (within her jurisdiction) }\end{array}$ \\
\hline $\begin{array}{c}\text { Competitive and integrated spot market } \\
\text { (represented by ISO) }\end{array}$ & $\begin{array}{c}\text { generation, demand, } \\
\text { power flow, nodal prices }\end{array}$
\end{tabular}

Table 1: Illustration of the three-stage game structure

\section{Stage III: A competitive spot market}

The third (bottom) level of the game is the competitive and integrated spot market, where generators, consumers, and TSOs of each zone take the network capacity as given for determining short-term dispatch and demand. The characteristics of the power network are taken into account using a loss-free direct-current load flow (DCLF) representation of Kirchhoff's laws, as is the current standard in this stream of modelling (e.g., Leuthold et al., 2012) 3 This approach explicitly accounts for the externalities occurring in meshed electricity systems ${ }^{4}$

Mathematically, the problem of a competitive market between generators and demand is equivalent to an Independent System Operator (ISO) optimizing welfare of the entire region (with the network taken as given), so we will use the latter notion in the mathematical derivation. Most importantly, in our subsequent formulation, congestion rents earned by TSOs are based on actual flows rather than financial transmission rights to properly account for these externalities.

\footnotetext{
${ }^{3}$ The term DCLF is actually a misnomer, as it is not based on the assumption of direct-current electricity flows; instead, it is a linearization of the non-convex alternating-current (AC) power flow model.

${ }^{4}$ The current European market design for trading power across zone borders is based on auctions of transmission capacity, which are capped by net transfer capacity (NTC). Because power does not actually flow in such a way, the stated NTC values include security margins to ensure network feasibility in spite of loop flows. Oggioni and Smeers (2013) and Oggioni et al. (2012) discuss the difficulty of combining zonal pricing and trade based on net transfer capacities. The negative externalities arising out of NTC-based trade increase in lock-step with market integration and cross-border trade; European TSOs are currently looking into flow-based approaches to replace the NTC trading framework.
} 


\section{Stage II: The zonal planners}

The second (intermediate) stage is the Nash game between the zonal planners, each deciding on network upgrades in her jurisdiction. Every zonal planner aims to maximize welfare in her zone; congestion rents earned by the zonal TSO are explicitly included in the objective function. Rents from cross-border transmission and the expansion costs for these lines are shared equally between the adjacent zones.

The decision on cross-border line upgrades is not taken by the zonal planners, but by the player at the first stage of the game. This is the simplest setup for our illustrative model; we will return to potential modifications and more elaborate formulations of bargaining in the concluding section, when we discuss further research.

\section{Stage I: The supra-national coordination agency}

The first (top) level of our model is a supra-national coordination agency; she can represent, for example, the Agency for the Cooperation of Energy Regulators (ACER). While this player cannot influence the decision by the zonal planners on their domestic line upgrades, she can decide on the expansion of cross-border lines; because she anticipates the strategic reaction of zonal planners, she can set the cross-border line investment in such a ways as to induce them to end up in a second-best Nash equilibrium 5

Realizing that there may exist multiple equilibria in the game between the zonal planners (stage II), the coordination agency can therefore also be interpreted as an equilibrium selection mechanism - it coordinates among the zonal planners to make sure a "good" equilibrium is attained. The coordination agency has a central role in the mathematical implementation and the solution approach, which we will discuss in the next section.

\footnotetext{
${ }^{5}$ The first-best solution would be the supra-national network investment plan without strategic considerations by the zonal planners.
}

\begin{tabular}{|c|c|}
\hline \multicolumn{2}{|l|}{ Sets } \\
\hline$n \in N$ & ... nodes \\
\hline$s \in S$ & ... power plant unit, generation technology \\
\hline$r \in R$ & ... regulatory zone (price, TSO) \\
\hline$n \in N_{r}$ & $\ldots$ nodes in regulatory zone $r$ \\
\hline$l \in L$ & ... power lines \\
\hline$l \in L_{r}$ & $\ldots$ power lines in the jurisdiction of regulator $r$ \\
\hline \multicolumn{2}{|l|}{ Variables } \\
\hline$d_{n}$ & ... demand \\
\hline$g_{n s}$ & ... generation \\
\hline$p_{n}$ & ... locational marginal price \\
\hline$e_{l}$ & ... line expansion/investment \\
\hline \multicolumn{2}{|c|}{ Parameters } \\
\hline$\overline{g_{n s}}$ & ... generation capacity \\
\hline$c_{n s}^{G}$ & ... linear generation costs \\
\hline$c_{l}^{T}$ & ... linear transmission investment costs \\
\hline & ... fixed costs/guaranteed return for TSO \\
\hline$B_{n k}, H_{l k}$ & ... line/node impedance matrices \\
\hline
\end{tabular}

Table 2: Selected notation 


\section{Mathematical formulation}

We now turn to the mathematical formulation; Table 2 lists selected important notation used in this work; the remaining sets, variables and parameters are introduced where necessary. Our approach follows the methodology developed by Ruiz et al. (2012); we will discuss their approach and the extensions we introduce below, when we discuss the difficulties posed by multi-stage games ${ }^{6}$

\section{The Independent System Operator}

The ISO optimizes the short-term dispatch of power plants, deciding on the generation level $g_{n s}$ at each node $n$ and of technology $s$, demand $d_{n}$, and the voltage angle $\delta_{n}{ }^{7}$ Consumer welfare (utility from consuming electricity) is given by the function $\left(a_{n}-\frac{1}{2} b_{n} d_{n}\right) d_{n}$, while $c_{n s}^{G}$ are the per-unit (i.e., marginal) generation costs. From the point of view of the ISO, the line expansion $e_{l}$ is an exogenous parameter.

$$
\begin{aligned}
\max _{g, d, \delta} \sum_{n \in N}\left[\left(a_{n}-\frac{1}{2} b_{n} d_{n}\right) d_{n}-\sum_{s} c_{n s}^{G} g_{n s}\right] \\
\text { s.t. } \quad \sum_{s} g_{n s}-\sum_{k} B_{n k} \delta_{k}-d_{n}=0 \quad\left(p_{n}\right) \\
\bar{f}_{l}+e_{l}-\sum_{k} H_{l k} \delta_{k} \geq 0 \quad\left(\bar{\mu}_{l}\right) \\
\bar{f}_{l}+e_{l}+\sum_{k} H_{l k} \delta_{k} \geq 0 \quad\left(\underline{\mu}_{l}\right) \\
\bar{g}_{n s}-g_{n s} \geq 0 \quad\left(\beta_{n s}\right) \\
\delta_{\hat{n}}=0 \quad(\gamma) \\
g_{n s} \geq 0 \quad\left(\psi_{n s}\right) \\
d_{n} \geq 0 \quad\left(\phi_{n}\right)
\end{aligned}
$$

Constraint $1 \mathrm{~b}$ is the energy balance constraint; the dual variable to this is the price of energy at each node. Constraints $\sqrt{1 \mathrm{c}}-1 \mathrm{~d}$ ) are the thermal line capacity limits (in positive and negative power flow direction). The maximum available generation by node and technology is given by Constraint (1e). Using voltage angles to determine power flows requires the definition of a slack bus $(\hat{n})$, at which the voltage angle is 0 by assumption (Constraint $1 \mathrm{f}$ ). The dual variables for all constraints are given in brackets.

The ISO problem forms the lower stage to the optimization problem of the zonal planners, the subsequent game stage II; hence, the standard approach is to insert the ISO problem to

\footnotetext{
${ }^{6}$ A similar three-stage model is developed by Zerrahn and Huppmann $(2014)$; the authors use similar reformulations to the lowest-level equilibrium problem, derive Karush-Kuhn-Tucker (KKT) conditions of the intermediate strategic players and use the top-level player as an equilibrium selection mechanism. In their work, however, the intermediate (strategic) players are generators rather than zonal planners, seeking to maximize profits by strategically withholding generation capacity or intentionally congesting the network. The top-level player is the network planner facing a trade-off between investment costs and the welfareenhancing of additional line capacity; the welfare gains can be distinguished between efficiency gains (less congestion) and a higher degree of competition between strategic generators.

${ }^{7}$ This approach using voltage angles is equivalent to another method frequently used when formulating DCLF models, namely using power transmission distribution factors (PTDF).
} 
the upper-level problem as equilibrium constraints, specified below: 8

$$
\begin{aligned}
& c_{n s}^{G}-p_{n}+\beta_{n s}-\psi_{n s}=0 \quad \perp \quad g_{n s} \text { (free) } \\
& -a_{n}+b_{n} d_{n}+p_{n}-\phi_{n}=0 \quad \perp \quad d_{n} \text { (free) } \\
& \sum_{k} B_{k n} p_{k}+\sum_{l} H_{l n}\left(\bar{\mu}_{l}-\underline{\mu}_{l}\right)-\left\{\begin{array}{ll}
\gamma & \text { if } n=\hat{n} \\
0 & \text { else }
\end{array}\right\}=0 \quad \perp \quad \delta_{n} \text { (free) } \\
& \sum_{s} g_{n s}-\sum_{k} B_{n k} \delta_{k}-d_{n}=0 \quad \perp \quad p_{n} \text { (free) } \\
& \bar{f}_{l}+e_{l}-\sum_{k} H_{l k} \delta_{k} \geq 0 \quad \perp \quad \bar{\mu}_{l} \geq 0 \\
& \bar{f}_{l}+e_{l}+\sum_{k} H_{l k} \delta_{k} \geq 0 \quad \perp \quad \underline{\mu}_{l} \geq 0 \\
& \bar{g}_{n s}-g_{n s} \geq 0 \quad \perp \quad \beta_{n s} \geq 0 \\
& \delta_{\hat{n}}=0 \quad \perp \quad \gamma(\text { free }) \\
& g_{n s} \geq 0 \quad \perp \quad \psi_{n s} \geq 0 \\
& d_{n} \geq 0 \quad \perp \quad \phi_{n} \geq 0
\end{aligned}
$$

Gabriel and Leuthold (2010), as one example, formulate such a model by taking first-order KKT conditions of the ISO problem (1), and replace the complementarity (or slackness) constraints using disjunctive constraints (Fortuny-Amat and McCarl, 1981). This yields a mixed-integer problem; however, deriving stationarity conditions for the zonal planners requires to take the derivative of the objective function. When binary variables are included due to the disjunctive constraints formulation, this is not possible; hence, this method is not suitable for our approach. Another possibility to replace complementarity conditions was proposed by Siddiqui and Gabriel (2013), using Schur's decomposition and so-called SOS1-type variables. However, implementing this approach in a mathematically exact way also requires binary variables, and thus suffers from the same caveat.

Therefore, we apply the aforementioned methodology by Ruiz et al. (2012) as an alternative: we use strong duality to replace the optimization problem by a set of constraints, which can be included in the next stage's optimization problems without the need for binary variables.

All constraints of Problem (1) are linear and the objective function (1a) is concave (to be maximized); therefore, strong duality holds (for any non-trivial set of parameters that admits a feasible solution). The Lagrangian dual problem then reads as follows:

$$
\begin{gathered}
\min _{p, \bar{\mu}, \underline{\mu}, \beta, \gamma, \phi} \frac{1}{2} \sum_{n \in N} \frac{1}{b_{n}}\left(\left(a_{n}\right)^{2}+\left(p_{n}\right)^{2}+\left(\phi_{n}\right)^{2}-2 a_{n} p_{n}+2 a_{n} \phi_{n}-2 p_{n} \phi_{n}\right) \\
+\sum_{l \in L}\left(\bar{\mu}_{l}+\underline{\mu}_{l}\right)\left(\bar{f}_{l}+e_{l}\right)+\sum_{n \in N, s \in S} \beta_{n s} \bar{g}_{n s} \\
\text { s.t. } \begin{array}{rll}
c_{n s}^{G}-p_{n}+\beta_{n s}-\psi_{n s} & =0 & \left(g_{n s}\right) \\
-a_{n}+b_{n} d_{n}+p_{n}-\phi_{n} & =0 & \left(d_{n}\right)
\end{array} \\
\sum_{k} B_{k n} p_{k}+\sum_{l} H_{l n}\left(\bar{\mu}_{l}-\underline{\mu}_{l}\right)-\left\{\begin{array}{ll}
\gamma & \text { if } n=\hat{n} \\
0 & \text { else }
\end{array}\right\}
\end{gathered}
$$

${ }^{8}$ The equilibrium model $2 \mathrm{f}$ could be simplified by combining equations $2 \mathrm{a} / 2 \mathrm{~b}$ and equations $2 \mathrm{i} 2 \mathrm{j}$; however, we will require the more extensive version in the later reformulations. 
Strong duality holds, hence the objective values at the optimal solution of Problems (1) and (3) are identical. This can be stated formally in the following constraint:

$$
\begin{array}{r}
-\frac{1}{2} \sum_{n \in N} \frac{1}{b_{n}}\left(\left(a_{n}\right)^{2}+\left(p_{n}\right)^{2}+\left(\phi_{n}\right)^{2}-2 a_{n} p_{n}+2 a_{n} \phi_{n}-2 p_{n} \phi_{n}\right) \\
-\sum_{l \in L}\left(\bar{\mu}_{l}+\underline{\mu}_{l}\right)\left(\left(\bar{f}_{l}+e_{l}\right)-\sum_{n \in N, s \in S} \beta_{n s} \bar{g}_{n s}\right. \\
+\sum_{n \in N}\left[\left(a_{n}-\frac{1}{2} b_{n} d_{n}\right) d_{n}-\sum_{s} c_{n s}^{G} g_{n s}\right] \geq 0
\end{array}
$$

Strong duality entails that any vector $(g, d, \delta, p, \bar{\mu}, \mu, \beta, \gamma, \phi)$ satisfying the primal constraints $(1 \mathrm{~b} 1 \mathrm{~h}$ ), the dual constraints (3b 3d) as well as the strong-duality constraint (4) is an optimal solution to both the primal and the dual problem. We can now use this set of linear and quadratic constraints to replace the ISO optimality conditions in the optimization problems of the players at the higher stages of the game, without having to deal with complementarity constraints or binary variables due to using disjunctive constraints ${ }^{9}$

Normally when using strong duality, Constraint (4) would have to hold with equality; we write it as an inequality such that the quadratic constraint remains convex (for a given network, i.e., fixed $e_{l}$ ). Since the objective value of Problem (1) for any feasible solution is always smaller than the objective value of Problem (3) for any feasible solution due to weak duality, Constraint (4) can never hold with a strict inequality for a vector that is primal and dual feasible.

\section{The zonal planner}

The zonal planner $r$ aims to maximize the sum of generator profits, consumer surplus and congestion rent earned by the zonal TSO at all nodes within her zone $N_{r} \subset N$ by deciding on network upgrades for all lines $e_{l}$ within her jurisdiction; these are the lines in the set $L_{r} \subset L$. For cross-border lines, the investment level is taken as given by the zonal planner; both investment costs and congestions rents are shared equally between the two adjacent zonal TSOs by assumption. The parameter $s h r_{l r}$ denotes the share of costs and rents of line $l$ to be attributed to the TSO of zone $r$; it is 1 for domestic lines, 0.5 for cross-border lines, and 0 otherwise.

\footnotetext{
${ }^{9}$ The constraints to the dual problem $\sqrt{3 \mathrm{~b}} 3 \mathrm{~d}$ ) are also the stationarity (KKT) conditions, if one would solve this problem as an equilibrium problem (or mathematically, a mixed complementarity problem, MCP), as given by Equations 2 . The solution vector of the strong duality problem would also solve the MCP, and vice versa.
} 
Mathematically, the optimization problem of the zonal planners reads as follows:

$$
\begin{aligned}
\max _{e_{l} \in L_{r}} \sum_{n \in N_{r}}\left[\left(a_{n}-\frac{1}{2} b_{n} d_{n t}-p_{n}\right) d_{n}+\sum_{s}\left(p_{n} g_{n s}-c_{n s}^{G} g_{n s}\right)\right] & \\
-\sum_{l \in L_{r}}\left[s h r_{l r}\left(\sum_{k} H_{l k} \delta_{k}\right)\left(\sum_{k} I_{l k} p_{k}\right)-\sum_{l \in L_{r}} c_{l}^{T} e_{l}\right] & \\
\text { s.t. } c_{n s}^{G}-p_{n}+\beta_{n s}-\psi_{n s}=0 & \left(\eta_{n s r}^{R}\right) \\
-a_{n}+b_{n} d_{n}+p_{n}-\phi_{n}=0 & \left(\rho_{n r}^{R}\right) \\
\sum_{k} B_{k n} p_{k}+\sum_{l} H_{l n}\left(\bar{\mu}_{l}-\underline{\mu}_{l}\right)-\left\{\begin{aligned}
& \gamma \text { if } n=\hat{n} \\
& 0 \quad \text { else }
\end{aligned}\right\}=0 & \left(\nu_{n r}^{R}\right) \\
\sum_{s} g_{n s}-\sum_{k} B_{n k} \delta_{k}-d_{n}=0 & \left(\lambda_{n r}^{R}\right) \\
\bar{f}_{l}+e_{l}-\sum_{k} H_{l k} \delta_{k} \geq 0 & \left(\bar{\mu}_{l r}^{R}\right) \\
\bar{f}_{l}+e_{l}+\sum_{k} H_{l k} \delta_{k} \geq 0 & \left(\underline{\mu}_{l r}^{R}\right) \\
\bar{g}_{n s}-g_{n s} \geq 0 & \left(\beta_{n s r}^{R}\right) \\
\delta_{\hat{n}}=0 & \left(\gamma_{r}^{R}\right) \\
g_{n s} \geq 0 & \left(\psi_{n s r}^{R}\right) \\
d_{n} \geq 0 & \left(\phi_{n r}^{R}\right) \\
-\sum_{n \in N}\left[\left(a_{n}-\frac{1}{2} b_{n} d_{n}\right) d_{n}-\sum_{s} c_{n s}^{G} g_{n s}\right] \geq 0 & \left(\kappa_{r}^{R}\right) \\
\sum_{n \in N} \frac{1}{b_{n}}\left(\left(a_{n}\right)^{2}+\left(p_{n}\right)^{2}+\left(\phi_{n}\right)^{2}-2 a_{n} p_{n}+2 a_{n} \phi_{n}-2 p_{n} \phi_{n}\right) & \\
\sum_{l \in L}\left(\bar{\mu}_{l}+\underline{\mu}_{l}\right)\left(\left(\bar{f}_{l}+e_{l}\right)-\sum_{n s} \bar{g}_{n s}\right. & \\
+\beta_{n \in N \in S} &
\end{aligned}
$$

Constraints $5 \mathrm{~b} 51$ are the primal and dual constraints from the ISO (lower-level) problem and the strong-duality constraint; they ensure optimality of the ISO lower-level problem given the network investment decision variables.

\section{The Nash game between zonal planners}

The optimization problem faced by one zonal planner (Problem 5) is similar to the model presented by Buijs and Belmans (2011); since the lower-level is an equilibrium problem, each zonal planner faces an MPEC. Searching for Nash equilibria between several MPECs, we obtain an EPEC. The constraints faced by each zonal planner are identical; nevertheless, the valuation that the zonal planner accords to them (i.e., the dual variables marked with an $R$ superscript) may differ across the planners. Therefore, the problem at hand is a Generalized Nash game (GNE). Harker (1991) proposes to assign identical duals to all shared constraints by assumption; this facilitates the solution of such problems considerably, since the problem then reduces to a Nash equilibrium (NE), which can be solved as a Variational Inequality (VI) or Mixed Complementarity Problem (MCP) using standard methods. This assumption can be interpreted as an implicit auction of the scarce resource or constraining factor in some 
cases; there exists a coordination mechanism between the players affected by the shared constraint. However, since one of the main tenets of our work is that such a coordination mechanism does not exist, we do not use this approach here. Instead, we will present a reformulation based on disjunctive constraints below to circumvent this problem without having to make a-priori assumptions.

We proceed by taking first-order (KKT) conditions of the zonal planner's MPEC, stated in the Appendix; however, both the objective function (5a) and the strong-duality constraint (51) are non-convex, because the line expansion is now a variable. As a consequence, the KKT conditions are neither necessary nor sufficient for an equilibrium, and there may be a multitude of KKT points. Let us first discuss the caveat that the KKT conditions are not necessary: this means that there may exist equilibria of the game between zonal planners that our approach does not capture. Mathematically, such equilibria are related to relaxed definitions of the Nash equilibrium, such as a Nash Bouligand stationary point (or Nash B-stationary point, Kulkarni and Shanbhag, 2013). In our defense, it is not clear whether different approaches such as enumeration or diagonalization would be able to identify all equilibria in applied problems.

The caveat that KKT conditions are not sufficient for an equilibrium is, in contrast, easy to overcome. We treat each KKT point as a candidate equilibrium and check for each zonal planner whether a profitable deviation exists, keeping all line capacities not within the jurisdiction of the respective zonal planner fixed. The algorithm is explained in more detail below, when we summarize the methodological advances of our approach. If no profitable deviation exists for any zonal planner, we keep the KKT point as an equilibrium; otherwise, we discard it. Our approach therefore only admits global Nash equilibria, in contrast to other approaches that only focus on deviation incentives in the neighbourhood of equilibrium candidates (local Nash equilibrium, $\mathrm{Hu}$ and Ralph, 2007).

In this way, we can use the KKT conditions as constraints to describe strong global Nash equilibria; the decision which equilibrium to choose is taken by the top-level player in this game, the supra-national planner.

\section{The supra-national planner}

The supra-national planner decides on cross-border network investment, anticipating the optimal strategic reaction of the zonal planners. She seeks to maximize welfare in the entire region, less the required investment costs:

$$
\begin{aligned}
& \max _{g, d, \delta} \sum_{n \in N}\left[\left(a_{n}-\frac{1}{2} b_{n} d_{n}\right) d_{n}-\sum_{s} c_{n s}^{G} g_{n s}\right]-\sum_{l \in L} c_{l}^{T} e_{l} \\
& \text { s.t. } \quad \text { equilibrium between the zonal regulators (KKT conditions 9) } \\
& \quad \text { and optimal dispatch by the ISO }
\end{aligned}
$$

As already mentioned above, there may exist multiple Nash equilibria. We therefore implement an iterative algorithm seeking to identify a range of possible outcomes. This allows us to give a broader interpretation to the top-level player than Ruiz et al. (2012) have done: rather than just presenting one Nash equilibrium, we can compare several equilibria. We will return to this issue when discussing the numerical results.

\section{Methodological advances}

Our model extends the approach used by Ruiz et al. (2012) in three important aspects: firstly, they assume a piece-wise constant willingness-to-pay function of consumers, while we use a linear inverse demand curve. This allows to identify marginal benefits of network expansion. 
Secondly, Ruiz et al. hold the dual variable associated with lower-level complementarity $\left(\kappa^{R}\right.$ in our formulation) fixed and test for different (exogenous) values.

The third contribution of our work relative to Ruiz et al. lies in the interpretation of the top level player: they use this player merely as an equilibrium selection mechanism, but do not discuss whether multiple Nash equilibria exist. We develop an iterative algorithm to identify alternative equilibria, and thus provide more room for interpretation of the threestage nature of the model.

\section{Reformulating the Generalized Nash game using disjunctive constraints}

One general methodological novelty (not discussed by Ruiz et al.) concerns the nature of the Generalized Nash Equilibrium (GNE) between the players at the intermediate model stage. In particular, as we mentioned earlier, we decide against the common simplification when solving GNE models of assuming identical multipliers for shared constraints (Harker, 1991) or a fixed, exogenously specified ratio between the multipliers (Oggioni et al., 2012). Instead, we replace the complementarity constraints of the zonal planners' and the ISO's KKT conditions by disjunctive constraints. This allows us to circumvent the problem of a non-square MCP model: we illustrate the approach using the example of the nodal energy balance faced by the ISO (constraint 1e).

$$
\bar{g}_{n s}-g_{n s} \geq 0
$$

For optimality, either this constraint is not binding, or the associated dual variable for the ISO $\left(\beta_{n s}\right)$ equals 0 . However, each zonal planner must also consider this constraint in her own optimization problem (Constraint 5h), with the associated dual variable $\left(\beta_{n s r}^{R}\right)$. The entire problem then reads as follows:

$$
\begin{array}{lll}
\bar{g}_{n s}-g_{n s} \geq 0 & \perp & \beta_{n s} \\
\bar{g}_{n s}-g_{n s} \geq 0 & \perp & \beta_{n s r}^{R}
\end{array} \quad \forall r \in R
$$

It is not possible to solve such a model with shared constraints as an MCP, because it is essentially a non-square system (more variables than unique equations). However, we can replace the entire system using disjunctive constraints. We introduce one large scalar $K$ and a binary variable $r_{n s}$ for each constraint.

$$
\begin{aligned}
\bar{g}_{n s}-g_{n s} & \geq 0 \\
\bar{g}_{n s}-g_{n s} & \leq K r_{n s} \\
\beta_{n s}+\sum_{r} \beta_{n s r}^{R} & \leq K\left(1-r_{n s}\right)
\end{aligned}
$$

This reformulation allows to include shared constraints without the requirement to make assumptions on the ratio of the dual variables of the shared constraints between the different players. For shared equality constraints, the complementarity condition can be neglected altogether. We reformulate all inequality constraints of the zonal planners' KKT conditions (stated in the Appendix) in this way. After the reformulation, we can also eliminate the strong-duality constraint (51): the primal and dual feasibility of the ISO problem is already included in the zonal planners' constraints, and complementarity of the ISO's variables is enforced by the disjunctive constraints reformulation. After this simplification, the resulting problem is only bilinear in the dual variable to the strong duality constraint $\left(\kappa_{r}^{R}\right)$.

\section{Optimistic vs. pessimistic bilevel games}

There is one general concern of modelling bilevel games that we still need to address: when solving two-stage games, the most commonly applied approach uses first-order optimality condition of the lower-level optimization problems and inserts those as constraints to the 
upper-level optimization problem. An important assumption is implicitly taken here: the decision variables of the lower-level player are then, mathematically speaking, decision variables of the upper-level player, respecting optimality constraints. If the optimal response of the lower-level player is unique for any given decision of the upper-level player, this need not be a concern. However, there may be cases where uniqueness of the lower-level best response is not given; the lower-level player is indifferent between several options.

In such cases, the upper-level player may then decide which of the options the lower-level player "chooses". This is commonly referred to as the optimistic approach; it is the best the upper-level player can do given the bilevel game.

In contrast, one may consider a case where the lower-level player wants to do what is worst for the upper-level player, given a situation where she is indifferent between several options; this is the pessimistic approach. Such a situation may be plausible in cases where the upper- and lower-level players are rivals, and the lower-level player wants to maximize her profits - but also has an incentive to reduce the profits of the rival, if it does not have a downside for her (by definition, own payoff is not affected, as she is indifferent between two decisions). Mathematically, this leads to a min-max-problem, which is computationally difficult to solve.

In the three-stage model (Problem 6), we implicitly use the optimistic approach, where optimistic is to be seen from the supra-national planner's point of view. We believe that this makes sense: the supra-national planner and the ISO's objective are well aligned. For the zonal planners, we assume that they are only concerned with national welfare - but we do not presume that they are malevolent towards other states/zones.

For the deviation-incentive checking algorithm (explained in the following section), we solve the optimization model of the zonal planners (Problem 5); here, we also use the optimistic approach (now optimistic from the point of view of the respective zonal planner). Again, this makes sense, because our question when solving this model is to check whether the zonal planner has a deviation incentive from the equilibrium candidate solution identified by the three-stage model. If we do no find a deviation incentive under the optimistic assumption, we can be confident that indeed there is none 10

\section{An iterative solution algorithm to identify multiple equilibria}

Let us now turn to the iterative algorithm to identify multiple equilibria, which we already referred to earlier. The problem of our three-stage model is as follows: there may exist multiple equilibria in the national-strategic game, and there may be KKT points which are not stable in the Nash sense, because a zonal planner finds a profitable deviation - in this case, the KKT conditions would hold because it is a local welfare minimum or saddle point in one or more variables of a zonal planner. This section describes an iterative algorithm to identify multiple equilibria, and to check for deviation incentives:

1 Solve the national-strategic model (Problem 6). The optimal line expansion is denoted as $e_{l}^{*}(i)$, where $i$ is the iteration counter; let the set $I$ collect all iterations of this algorithm.

2 Loop over zonal planners: Solve the zonal MPEC problem (Problem 5), with all line investments but the domestic ones fixed to the level determined in step $1, e_{l}=e_{l}^{*}(i) \forall l \notin$ $L_{r}$. If at least one zonal planner has a profitable deviation, discard the KKT point as an equilibrium candidate.

\footnotetext{
${ }^{10}$ We are aware that this assumption may exclude equilibria in the game between zonal planners, which are not deviation-proof under the optimistic assumption, but are incentive-compatible if the ISO can credibly commit to "retaliate" by following the pessimistic strategy. However, identifying whether such a case may even exist is beyond the scope of this work.
} 
3 If the KKT point identified is an equilibrium candidate: check whether a previously found equilibrium dominates the current candidate solution. Definition: a solution $A$ dominates another solution $B$ if each zonal planner is (weakly) better off in solution $A$ than in $B$. If no dominating previous solution is found, keep this KKT point as an equilibrium.

4 Add constraints to the national-strategic model (Problem 6) such that the solution $e_{l}^{*}(i)$ is excluded from the feasible region. To this end, we introduce auxiliary binary variables $z_{l}^{+}(i), z_{l}^{-}(i)$; the parameter $\overline{e_{l}}$ is an suitable large upper bound on the line expansion:

$$
\left.\begin{array}{l}
e_{l}>z_{l}^{+}(i) e_{l}^{*}(i) \\
e_{l}<\overline{e_{l}}-z_{l}^{-}(i)\left(\overline{e_{l}}-e_{l}^{*}(i)\right) \\
\quad \sum_{l} z_{l}^{+}(i)+z_{l}^{-}(i) \geq 1
\end{array}\right\} \quad \forall i \in I
$$

The logic is straightforward: at least one of the auxiliary binary variables must equal 1 for each previous iteration; hence, these constraints ensure the next KKT point found in Problem (6) differs with regard to at least one line expansion variable from all previously found KKT points. This must hold irrespective of whether a point was a stable equilibrium or not 11

\section{... Repeat at step 1 ; stop after $n$ iterations}

There is one important methodological and conceptual benefit of this iterative solution to the more "hands-on" approach of solving such a game by diagonalization and attempting to find different equilibria by using various starting points: using diagonalization, one can make no informed statement whether "better" equilibria exist, in the sense that there is a dominating equilibrium (Definition in Step 3 of the iterative algorithm). In contrast, using our iterative algorithm, the first KKT point will be the candidate equilibrium with the highest level of aggregate welfare - irrespective of whether this point is an equilibrium. The second iteration will provide the KKT point with the second-highest overall welfare, and so forth. As we stated earlier, there may exist equilibria which are not KKT points our approach cannot find these, so the above statement does not apply to such equilibria. However, our approach is at least an improvement to the diagonalization approach 12

\section{Solution strategy and two benchmark models}

The three-stage model is, after the disjunctive constraints reformulation, a quadratic program with mixed-integer non-convex constraints. We implement the problem in GAMS and use the BARON solver (Tawarmalani and Sahinidis, 2005); this solver aims to solve non-convex problems to global optimality.

In order to identify and quantify the impact of strategic zonal planners, we compute two benchmark cases: first, a model without any investment at all, which is exactly the ISO dispatch problem (Problem 1). As a second benchmark, we compute the optimal supranational investment problem without strategic consideration by zonal planners. This can be computed by combining the objective function of the supra-national planner (6a) with the operational constraints of the ISO problem (Constraints $1 \mathrm{~b} 1 \mathrm{~h}$ ), allowing investments on all lines. Both benchmark models are convex quadratic optimization problems (QP) and are numerically solved using the CPLEX solver.

\footnotetext{
${ }^{11}$ Otherwise, the iterative algorithm would get stuck at this point. In the GAMS implementation, we use an $\epsilon$ distance to replace the strict by a weak inequality.

${ }^{12}$ For further research, we intend to attempt reformulations such that the KKT conditions of the zonal planners are necessary; in this case, our iterative approach would indeed identify all equilibria, ranked by aggregate welfare. Of course, our statements only hold under the assumption that the BARON solver identifies the correct global optimum of the non-convex problem.
} 


\section{An illustrative example}

We apply this model to a four-node, two-country example, which is motivated by the current situation between Germany and Poland. We assume two countries with two nodes each; the south of country A has a large industrial base, while the north of country A has only low demand for electricity. Country B has intermediate demand at both nodes. The energy system of both countries used to be based on autarky of each zone, with limited transmission capacity between them.

Two shifts caused a significant imbalance in the power network: large installation of generation capacity with low variable costs in the north of Country A (e.g., wind power in the coastal region), and decommissioning of base-load generation capacity in the south of Countries A and B (e.g., the nuclear phase-out in Germany, less power generation from coal due to emission reduction efforts). We abstract from investment in power generation; we assume that the shifts in the generation portfolio have already taken place, but not at the locations where it would be optimal from the point of view of the entire system. This can be interpreted as the effects caused by fixed feed-in tariffs or other subsidies for renewables; in some cases, renewable support does not give sufficient consideration to locational aspects. The decommissioning of nuclear power plants is also a political decision rather than based on the short-run economics of power markets.

Figure 1 depicts the network, the installed generation capacity by type, the reference demand at each node, the initial transmission capacity, and the equilibrium prices in the absence of network expansion. All quantities, flows and capacities presented here are given in Gigawatthours (GWh) ${ }^{13}$, all prices and costs are given in Euro per Megawatthour (MWh). We assume three types of power plants: "renewables" with zero marginal generation costs, "base" with $40 € /$ MWh and "peak" with $70 € /$ MWh. The parameters for the inverse demand function are computed using a price of $70 € / \mathrm{MWh}$ and an elasticity of -0.25 . The costs for network expansion are assumed to be $2 € / M W h{ }^{14}$ The impedance of all lines is assumed to be identical.

Without substantial upgrades of the transmission grid, we observe a large price differential between the two nodes of Country A. However, in order to transmit power to the node with the highest willingness-to-pay, upgrades must also be made in the northern cross-border line and the line within Country B due to the particular power flow characteristics in an alternating-current $(\mathrm{AC})$ power grid.

We first compute two benchmark cases: no investment and (supra-national) welfareoptimal investment. In the initial (current) network, peak-load generators in both southern nodes are the price-setting plants - there is idle capacity at node $n 1$, but it cannot be transported to the high-price nodes due to the constraints of the network. With welfareoptimal investment in transmission capacity, prices in node $n 2$ slightly decrease, as more base-load generation can be shipped to that node. Prices in the northern nodes increase in line with a general price convergence - the resulting price differential between the nodes is exactly equal to the costs of building an additional marginal unit of line capacity.

Because the plant in node $n 4$ is still the price-setting plant in the system even with welfare-optimal line expansions, prices converge towards the high price at that node; this may seem counter-intuitive at first given that network investment is generally expected to exhibit a price-decreasing effect. Nevertheless, welfare is increased due to the network expansion, because peak-load (i.e., high variable cost) generation can be replaced by the base-load plant at node $n 1$ which was idle in the no-investment benchmark case.

\footnotetext{
${ }^{13}$ Capacities are actually in Gigwatt $(\mathrm{GW})$ rather than GWh (energy). By assuming one representative hour, the two are equivalent.

${ }^{14}$ This value is derived from the following assumptions: a double-circuit $380 \mathrm{kV}$ line costs 1.4 million $€ / \mathrm{km}$; the distance between two nodes is $400 \mathrm{~km}$; the line is used at full capacity in half of the hours every year; the lifetime of the line is 40 years; and the interest rate is $4 \%$ p.a.
} 
Benchmark: No investment

\begin{tabular}{|c|c|}
\hline \multicolumn{2}{|l|}{ Node $n 1$} \\
\hline \multicolumn{2}{|l|}{ Generation } \\
\hline Renewables & $20 \quad(20)$ \\
\hline Base & $22.21(40)$ \\
\hline Load & $33.21(30)$ \\
\hline \multicolumn{2}{|l|}{ Line $l 1$} \\
\hline \multicolumn{2}{|l|}{ Capacity } \\
\hline \multicolumn{2}{|l|}{ Node $n \mathscr{2}$} \\
\hline \multicolumn{2}{|l|}{ Generation } \\
\hline Base & $(30)$ \\
\hline Peak & (40) \\
\hline Load & $(50)$ \\
\hline
\end{tabular}

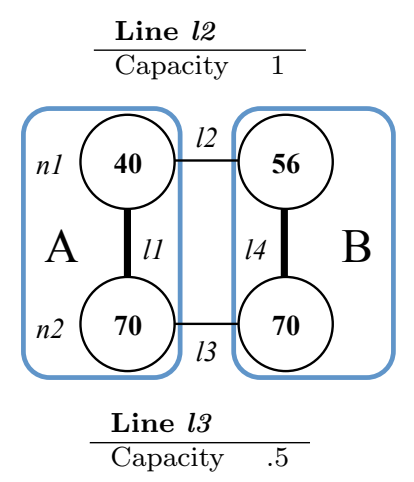
Node $n 3$
Generation
Base $\quad 30$
(30)
Load $21 \quad(20)$
Line 14
Capacity 10

Node $n 4$

$\begin{array}{lll}\begin{array}{l}\text { Generation } \\ \text { Peak }\end{array} & 9 & (25) \\ \text { Load } & 20 & (20)\end{array}$

$20 \quad(20)$

Benchmark: Welfare-optimal investment

\begin{tabular}{llr} 
Node $\boldsymbol{n} \mathbf{1}$ & & \\
\hline Generation & & \\
Renewable & 20 & $(20)$ \\
Base & 40 & $(40)$ \\
\hline Load & $30.43(30)$ \\
Line $\boldsymbol{l} \mathbf{1}$ & & \\
\hline Capacity & $10+\mathbf{1 2 . 3 4}$ \\
Node $\boldsymbol{n} \mathbf{2}$ & & \\
\hline Generation & & \\
Base & 30 & $(30)$ \\
Peak & 0 & $(40)$ \\
\hline Load & $50.36(50)$
\end{tabular}

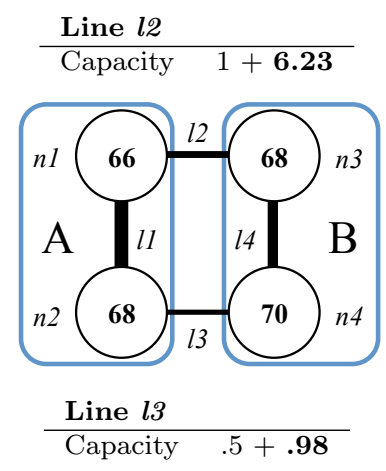

\begin{tabular}{|c|c|}
\hline \multicolumn{2}{|l|}{ Node $n 3$} \\
\hline \multicolumn{2}{|l|}{ Generation } \\
\hline Base & $(30)$ \\
\hline Load & $20.14(20)$ \\
\hline \multicolumn{2}{|l|}{ Line 14} \\
\hline Capacity & $10+\mathbf{7 . 0 9}$ \\
\hline \multicolumn{2}{|l|}{ Node $n 4$} \\
\hline \multicolumn{2}{|l|}{ Generation } \\
\hline Peak & $0.93(25)$ \\
\hline Load & $(20)$ \\
\hline
\end{tabular}

Figure 1: Benchmark cases in the illustrative two-zone, four-node network; generation capacity and reference load in brackets; investment in bold; nodal prices given in the circles in the figure 


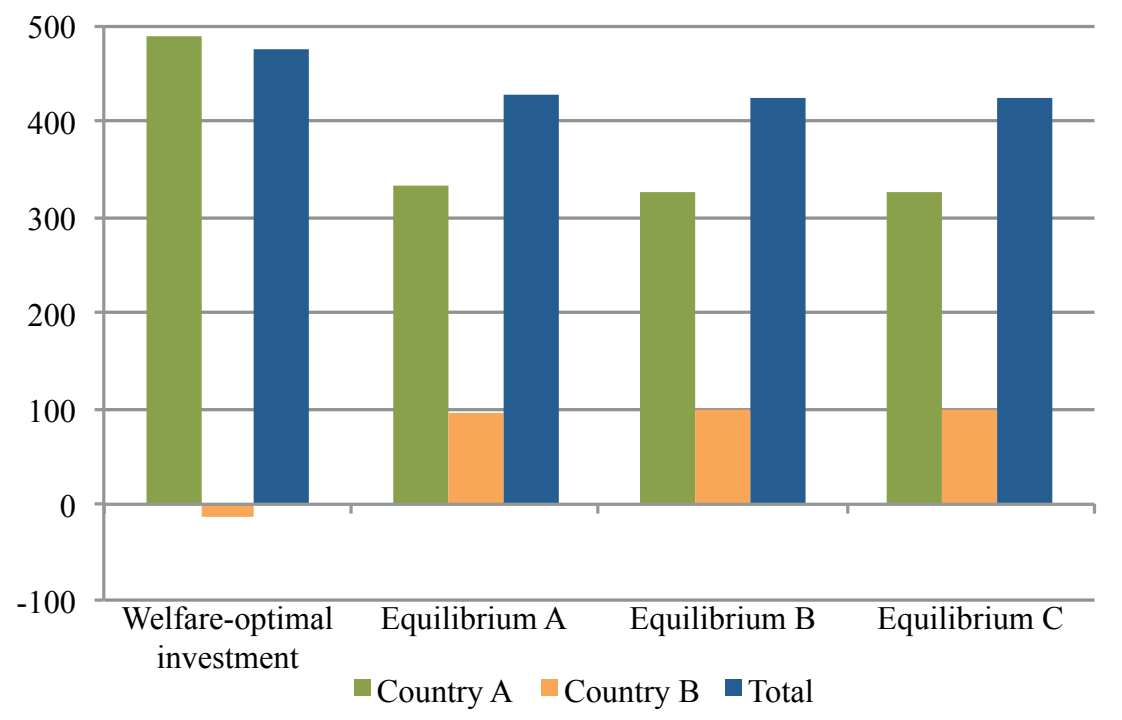

Figure 2: Welfare gains relative to the No investment benchmark in $1000 €$

However, when analysing the welfare shifts due to the network expansion as illustrated in Figure 2 and Table 3 , one notices that all welfare gains accrue in Country A, while Country B finds her welfare decreased relative to the status quo. This begs the question whether a zonal planner would not find it in her best interest to restrict the network expansion within her jurisdiction, since explicit transfers between the zones is not possible in the current setting.

To investigate this effect, we compute equilibria of the three-stage game; we use the iterative algorithm presented in Section 4 to identify $46 \mathrm{KKT}$ points of the game ${ }^{15}$ Of these 46 candidate solutions, 5 are not Nash equilibria (i.e., at least one of the zonal planner identified a profitable deviation). Another $35 \mathrm{KKT}$ points are Nash equilibria in the sense that no zonal planner has a unilateral deviation incentive, but the equilibria are not Pareto efficient (also referred to as Pareto optimal). A Pareto improvement exists; the solutions are dominated by other equilibria. ${ }^{16}$ Three iterations experienced numerical difficulties and where therefore excluded. This leaves three Nash equilibria found at iterations 1, 23 and 27; they are presented in Figure 3.

In general, network expansion is lower than in the welfare-optimal benchmark. The difference is most pronounced on line $l 4$, where expansion is lower by one third relative to the benchmark. This makes sense intuitively: the zonal planner of country B specifically "withholds" line expansion; this has the effect to reduce prices at node $n 3$ and thereby shifts rents from generators at node $n 1$ to the consumers at node $n 3$. Equilibrium $\mathrm{C}$ is the only solution where not all lines are fully congested; this is line $l 3$, which is consequently not expanded.

In the three national-strategic equilibria, aggregate welfare is $10 \%$ lower compared to the welfare-optimal benchmark (see Table 3). At the same time, by strategically reducing line expansion on the domestic line, the zonal planner of country $B$ is able to appropriate

\footnotetext{
${ }^{15}$ Due to the "holes" cut into the feasible region by the iterative algorithm to identify other KKT points, the solver requires more time after each iteration. The $47^{\text {th }}$ iteration was aborted after 24 hours of computation time.

${ }^{16} \mathrm{~A}$ dominated equilibrium is one where another equilibrium was found which is a welfare improvement for all zonal planners. Thus, agreeing to move to the dominating equilibrium is in every player's best interest; a joint deviation incentive for all players without transfers/compensation exists.
} 
Equilibrium $A$ - Iteration 1

\begin{tabular}{llr} 
Node $\boldsymbol{n} \mathbf{1}$ & & \\
\hline Generation & & \\
Renewables & 20 & $(20)$ \\
Base & 40 & $(40)$ \\
\hline Load & $32.05(30)$ \\
Line $\boldsymbol{l 1}$ & & \\
\hline Capacity & $10+$ & $\mathbf{1 1 . 3 9}$ \\
Node $\boldsymbol{n} \boldsymbol{2}$ & & \\
\hline Generation & & \\
Base & 30 & $(30)$ \\
Peak & 0 & $(40)$ \\
\hline Load & $51.71(50)$
\end{tabular}

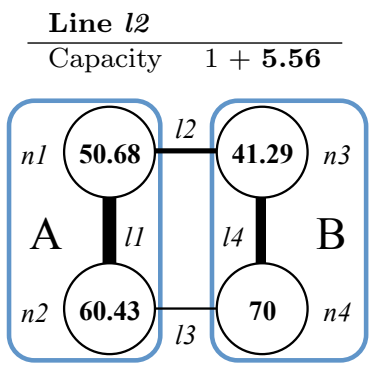

Line 13

Capacity $\quad .5+\mathbf{0 . 3 7}$

\begin{tabular}{lll} 
Node $\boldsymbol{n 3}$ & & \\
\hline $\begin{array}{l}\text { Generation } \\
\text { Base }\end{array}$ & $30 \quad(30)$ \\
\hline Load & $22.05(20)$
\end{tabular}

Line $l 4$

Capacity $10+\mathbf{4 . 5 1}$

Node $n_{4}$

Generation

Peak $5.81(25)$

Load $20 \quad(20)$

Equilibrium $B$ - Iteration 23

Node $n 1$

Generation

Renewable $20 \quad(20)$

\begin{tabular}{clr} 
Base & $40 \quad(40)$ \\
\hline Load & $32.12(30)$
\end{tabular}

Line $l 1$

Capacity $10+\mathbf{1 1 . 3 5}$

\begin{tabular}{lrr} 
Node $\boldsymbol{n} \boldsymbol{2}$ & & \\
\hline Generation & & \\
Base & 30 & $(30)$ \\
Peak & 0 & $(40)$ \\
\hline Load & $51.77(50)$
\end{tabular}

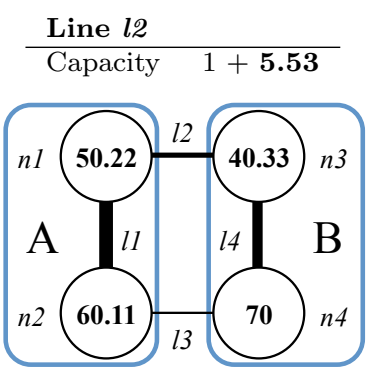

Line 13

Capacity $.5+.47$
Node $n 3$

Generation

Base $\quad 30 \quad(30)$

Load $22.12(20)$

Line $l 4$

Capacity $10+\mathbf{4 . 4 1}$

Node $n 4$

Generation

Peak

$6 \quad(25)$

Load $20 \quad(20)$

\section{Equilibrium $C$ - Iteration 27}

\begin{tabular}{llr} 
Node $\boldsymbol{n} \mathbf{1}$ & & \\
\hline $\begin{array}{l}\text { Generation } \\
\text { Renewable }\end{array}$ & 20 & $(20)$ \\
Base & 40 & $(40)$ \\
\hline Load & $32.14(30)$ \\
Line $\boldsymbol{l} \mathbf{1}$ & & \\
\hline Capacity & $10+$ & $\mathbf{1 1 . 3 4}$ \\
Node $\boldsymbol{n} \mathbf{2}$ & & \\
\hline Generation & & \\
Base & 30 & $(30)$ \\
Peak & 0 & $(40)$ \\
\hline Load & $51.79(50)$
\end{tabular}

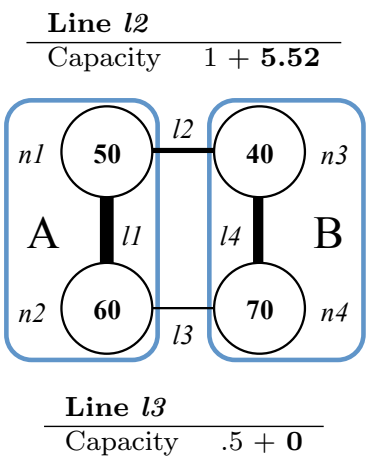

\begin{tabular}{ll} 
Node $\boldsymbol{n 3}$ & \\
\hline $\begin{array}{l}\text { Generation } \\
\text { Base }\end{array}$ & $30 \quad(30)$ \\
\hline Load & $22.14(20)$ \\
Line $l 4$ & \\
\hline Capacity & $10+\mathbf{4 . 3 8}$ \\
Node $\boldsymbol{n 4}$ & \\
\hline $\begin{array}{l}\text { Generation } \\
\text { Peak }\end{array}$ & $6.07(25)$ \\
\hline Load & $20 \quad(20)$
\end{tabular}

Figure 3: Three-stage game equilibria in the illustrative two-zone, four-node network; generation capacity and reference load in brackets; investment in bold; nodal prices given in the circles in the figure 
more than $20 \%$ of the welfare gains, rather than seeing her constituents worse off due to the network expansion.

As a last remark, let us compare the three equilibria: it is clear that Equilibrium A is the network expansion with the highest aggregate welfare. However, one may argue that Equilibrium C is the more "equitable" outcome from the point of view of the supra-national planner, as both zones benefit more evenly from the expansion. In any case, the difference between the three equilibria is relatively small. We are not sure whether this is due to the small scale of the sample network, an artefact arising from the numerical aspects of the non-convex problem, or an inherent property of the game structure.

\section{Conclusions and Outlook}

In a meshed power network, line expansions may lead to significant re-allocations of profits and rents across different stakeholder groups and national boundaries. Zonal (or national) interests and the lack of an efficient compensation mechanism may prevent reaping all potential welfare gains from network investments, because the beneficiaries of network expansion are located in different jurisdiction than those stakeholders bearing the costs. Zonal planners, such as governments, regulators, or national TSOs, may therefore have incentives to over-invest or intentionally withhold power line upgrades in their jurisdiction to induce a shift of rents towards their constituents. This may impede the efficient integration of the European energy market and the transformation towards a low-carbon power sector.

We develop a three-stage model to represent the Generalized Nash game between zonal planners, each taking into account how line capacity upgrades impacts the outcome in the competitive spot market. The game is led by a supra-national planner, who decides on crossborder line expansion. We make use of strong duality to replace the equilibrium constraints of the lowest-level player, the ISO managing the competitive spot market, and take first-order conditions of the zonal planners' optimization problem. Adapting a disjunctive constraints reformulation, we circumvent the common problem when solving Generalized Nash games, and do not need to make a priori assumptions on the relative valuations of shared constraints. Finally, we implement an iterative algorithm to determine multiple equilibria.

Our results based on a simple test case demonstrate that the national-strategic behaviour yields significant welfare loss compared to the system-optimal investment. A zonal planner has incentives to "under-invest" in her domestic line in order for her constituents to obtain a share of the welfare gains, rather than seeing her domestic welfare decreased by the systemoptimal network upgrades.

For further research and future model extensions, we intend to develop the three-stage approach in several directions: firstly, we want to relax the current simplification that all cross-border network investment is decided by the supra-national planner. Instead, those lines upgrades should be subject to a bargaining process between the adjacent zonal planners. This will require the explicit modelling of a compensation mechanism, in the spirit of the ITC and the "projects of common interest". It will be interesting to see whether an explicit transfer scheme can alleviate the failure to reach the first-best equilibrium.

As a second line of further research, we intend to focus on the welfare allocation between the different stakeholder groups, and the shifts between them due to network upgrades. One may imagine a situation where the regulator has a preference for the welfare of consumers, rather than the equal weight of consumer welfare, generator profits, and TSO rents, which we assumed so far. Furthermore, in such a setting, an explicit funding constraint of the TSO has to be considered; network usage tariffs will be used to finance additional transmission capacity. Thereby, we will be able to explicitly include the trade-off between the benefits of additional transmission capacity and the consumer welfare loss due to the tariffs. This is similar to the work by Daxhelet and Smeers (2007), but extends it for network investment. 


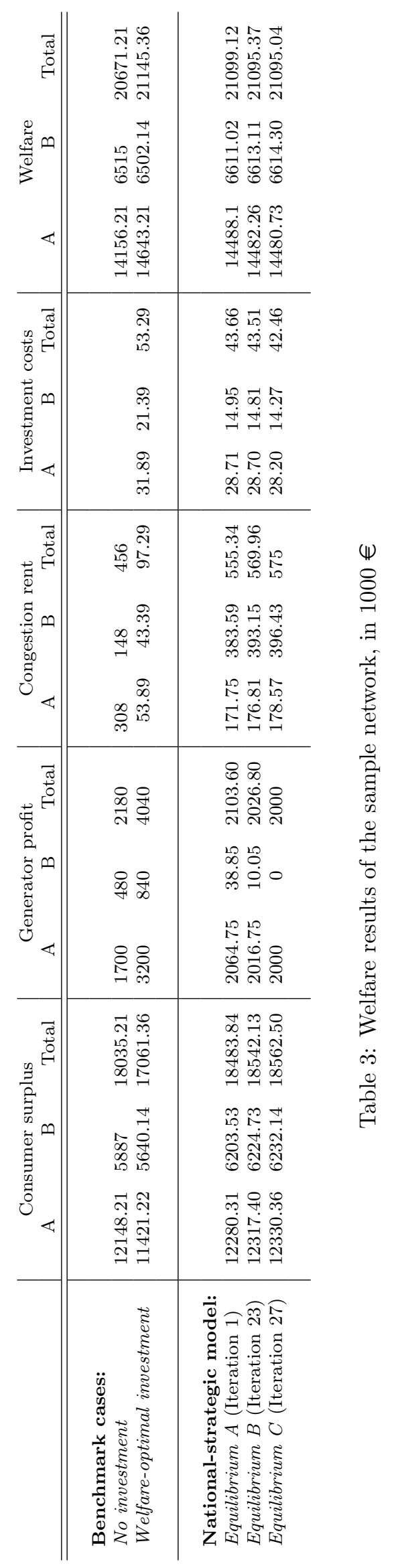




\section{References}

Francis Bloch and Matthew O. Jackson. Definitions of equilibrium in network formation games. International Journal of Game Theory, 34(3):305-318, 2006.

Francis Bloch and Matthew O. Jackson. The formation of networks with transfers among players. Journal of Economic Theory, 133(1):83-110, 2007.

Patrik Buijs and Ronnie Belmans. Transmission investments in a multilateral context. IEEE Transactions on Power Systems, 27(1):475-483, 2011.

Patrik Buijs, David Bekaert, and Ronnie Belmans. Seams issues in European transmission investments. The Electricity Journal, 23(10):18-26, 2010.

Olivier Daxhelet and Yves Smeers. The EU regulation on cross-border trade of electricity: A two-stage equilibrium model. European Journal of Operational Research, 181(3):1396$1412,2007$.

EC. Commission Regulation (EU) No 714/2009. European Commission, 2009.

EC. Commission Regulation (EU) No 838/2010. European Commission, 2010.

EC. Energy Roadmap 2050. Communication (2011) 885. European Commission, 2011.

EC. Making the internal energy market work. Communication (2012) 663 final . European Commission, 2012.

EC. Commission Regulation (EU) No 347/2013. European Commission, 2013.

Jonas Egerer and Hans Nylund. Regional versus bilateral cost sharing in electricity transmission expansion. http://pure.ltu.se/portal/files/78697853/Article.pdf, 2014.

Jonas Egerer, Clemens Gerbaulet, and Casimir Lorenz. European electricity grid infrastructure expansion in a 2050 context. DIW Discussion Paper 1299, 2013a.

Jonas Egerer, Friedrich Kunz, and Christian von Hirschhausen. Development scenarios for the North and Baltic Seas Grid - A welfare economic analysis. Utilities Policy, 27:123-134, 2013b.

ENTSO-E. The Ten-Year Network Development Plan and Regional Investment Plans. European Network Transmission System Operators - Electricity, 2013.

José Fortuny-Amat and Bruce McCarl. A Representation and Economic Interpretation of a Two-Level Programming Problem. The Journal of the Operational Research Society, 32 (9):783-792, 1981.

Steven A. Gabriel and Florian U. Leuthold. Solving discretely-constrained MPEC problems with applications in electric power markets. Energy Economics, 32(1):3-14, 2010.

Dermot Gately. Sharing the gains from regional cooperation: A game theoretic application to planning investment in electric power. International Economic Review, 15(1):195-208, 1974.

Patrick T. Harker. Generalized Nash games and quasi-variational inequalities. European Journal of Operational Research, 54(1):81-94, 1991.

Xinmin Hu and Daniel Ralph. Using EPECs to model bilevel games in restructured electricity markets with locational prices. Operations Research, 55(5):809-827, 2007. 
Ankur A. Kulkarni and Uday V. Shanbhag. Global equilibria of multi-leader multi-follower games. arXiv:1206.2968, 2013.

Thomas-Olivier Léautier and Véronique Thelen. Optimal expansion of the power transmission grid: why not? Journal of Regulatory Economics, 36(2):127-153, 2009.

Florian U. Leuthold, Hannes Weigt, and Christian von Hirschhausen. A Large-Scale Spatial Optimization Model of the European Electricity Market. Networks and Spatial Economics, 12(1):75-107, 2012.

Karsten Neuhoff, Julian Barquin, Maroeska G. Boots, Andreas Ehrenmann, Benjamin F. Hobbs, Fieke A.M. Rijkers, and Miguel Vázquez. Network-constrained Cournot models of liberalized electricity markets: The devil is in the details. Energy Economics, 27(3):495 525,2005 .

Hans Nylund. Regional cost sharing in expansions of electricity transmission grids. mimeo, 2013.

Giorgia Oggioni and Yves Smeers. Market failures of Market Coupling and counter-trading in Europe: An illustrative model based discussion. Energy Economics, 35:74-87, 2013.

Giorgia Oggioni, Yves Smeers, Elisabetta Allevi, and Siegfried Schaible. A generalized Nash equilibrium model of market coupling in the European power system. Networks and Spatial Economics, 12(4):503-560, 2012.

David Pozo, Javier Contreras, and Enzo E. Sauma. If you build it, he will come: Anticipative power transmission planning. Energy Economics, 36:135-146, 2013.

Juan Rosellón and Hannes Weigt. A dynamic incentive mechanism for transmission expansion in electricity networks: Theory, modeling, and application. The Energy Journal, 32 (1):119-148, 2011.

Sophia Rüster, Christian von Hirschhausen, Claudio Marcantonini, Xian He, Jonas Egerer, and Jean-Michel Glachant. EU involvement in electricity and natural gas transmission grid tarification. Think Report, European University Institute, 2012.

Carlos Ruiz, Antonio J. Conejo, and Yves Smeers. Equilibria in an oligopolistic electricity pool with stepwise offer curves. IEEE Transactions on Power Systems, 27(2):752-761, 2012.

Andreas Schröder, Thure Traber, and Claudia Kemfert. Market driven power plant investment perspectives in Europe: Climate policy and technology scenarios until 2050 in the model EMELIE-ESY. DIW Discussion Paper 1268, 2013.

Sauleh A. Siddiqui and Steven A. Gabriel. An SOS1-Based Approach for Solving MPECs with a Natural Gas Market Application. Networks and Spatial Economics, 13(2):205-227, 2013.

Mohit Tawarmalani and Nikolaos V. Sahinidis. A polyhedral branch-and-cut approach to global optimization. Mathematical Programming, 103(2):225-249, 2005.

Eckehard Tröster, Rena Kuwahata, and Thomas Ackermann. European Grid Study 2030/2050. energynautics GmbH, Germany, 2011.

Alexander Zerrahn and Daniel Huppmann. Network expansion to mitigate market power How increased integration fosters welfare. DIW Discussion Paper 1380, 2014. 


\section{Appendix}

The KKT conditions of the national regulator

$$
\begin{aligned}
& c_{l}^{T}-\left(\bar{\mu}_{l r}^{R}+\underline{\mu}_{l r}^{R}\right)+\kappa_{r}^{R}\left(\bar{\mu}_{l}+\underline{\mu}_{l}\right) \geq 0 \quad \perp \quad e_{l} \geq 0 \quad \text { if } l \in L_{r} \\
& {\left[-p_{n}+c_{n s}^{G}\right]_{\text {if } n \in N_{r}}} \\
& -\lambda_{n r}^{R}+\beta_{n s r}^{R}-\psi_{r n s}^{R}+\kappa_{r}^{R} c_{n s}^{G}=0 \quad \perp \quad g_{n s} \text { (free) } \\
& {\left[-a_{n}+b_{n} d_{n}+p_{n}\right]_{\text {if } n \in N_{r}}} \\
& -\rho_{n r}^{R} b_{n}+\lambda_{n r}^{R}-\phi_{n r}^{R}-\kappa_{r}^{R}\left(a_{n}-b_{n} d_{n}\right)=0 \quad \perp \quad d_{n} \text { (free) } \\
& {\left[\sum_{l \in L_{r}} \operatorname{sh} r_{l r} H_{l n} \sum_{k \in N} I_{l k} p_{k}\right]_{\text {if } n \in N_{r}}} \\
& +\sum_{k \in N} B_{k n} \lambda_{k r}^{R}+\sum_{l \in L} H_{l n}\left(\bar{\mu}_{l r}^{R}-\underline{\mu}_{l r}^{R}\right)-\left\{\begin{aligned}
\gamma_{r}^{R} & \text { if } n=\hat{n} \\
0 & \text { else }
\end{aligned}\right\}=0 \quad \perp \quad \delta_{n} \text { (free) } \\
& {\left[d_{n}-\sum_{s \in S} g_{n s}+\sum_{l \in L_{r}} s h r_{l r} I_{l n} \sum_{k \in N} H_{l k} \delta_{k}\right]_{\text {if } n \in N_{r}}} \\
& +\sum_{s \in S} \eta_{n s r}^{R}-\rho_{n r}^{R}-\sum_{k \in N} \nu_{k r}^{R} B_{n k}+\kappa_{r}^{R} \frac{1}{b_{n}}\left(p_{n}-a_{n}-\phi_{n}\right)=0 \quad \perp \quad p_{n} \text { (free) } \\
& -\sum_{n \in N} \nu_{n r}^{R} H_{l n}+\kappa_{r}^{R}\left(\bar{f}_{l}+e_{l}\right) \geq 0 \quad \perp \quad \bar{\mu}_{l} \geq 0 \\
& \sum_{n \in N} \nu_{n r}^{R} H_{l n}+\kappa_{r}^{R}\left(\bar{f}_{l}+e_{l}\right) \geq 0 \quad \perp \quad \underline{\mu}_{l} \geq 0 \\
& -\eta_{n s r}^{R}+\kappa_{r}^{R} \bar{g}_{n s} \geq 0 \quad \perp \quad \beta_{n s} \geq 0 \\
& \nu_{\hat{n} r}^{R}=0 \quad \perp \quad \gamma(\text { free }) \\
& \eta_{n s r}^{R} \geq 0 \quad \perp \quad \psi_{n s} \geq 0 \\
& \rho_{n r}^{R}+\kappa_{r}^{R} \frac{1}{b_{n}}\left(\phi_{n}+a_{n}-p_{n}\right) \geq 0 \quad \perp \quad \phi_{n} \geq 0
\end{aligned}
$$

Equations 5b-51] in complementarity form

As explained in Section 4 the strong duality constraint is replaced by the ISO's equilibrium constraints (Equations 21. All complementarity conditions are replaced by the disjunctive constraints reformulation. The constraints are bilinear (and non-convex) in the dual variable to the strong-duality constraint $\left(\kappa_{r}^{R}\right)$. 